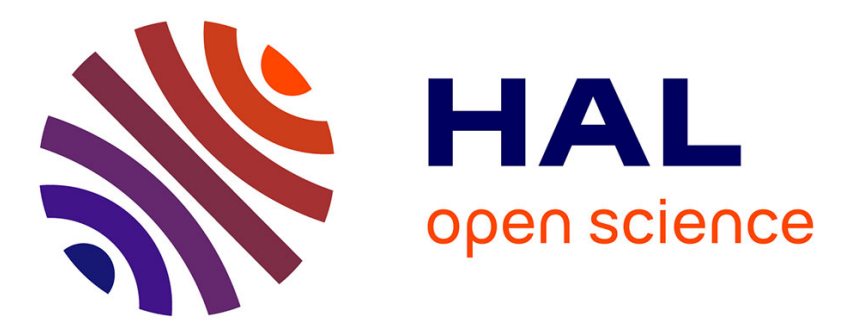

\title{
Opportunistic Feedback Reporting and Scheduling Scheme for Multichannel Wireless Networks
}

\author{
Matha Deghel, Mohamad Assaad, Mérouane Debbah
}

\section{To cite this version:}

Matha Deghel, Mohamad Assaad, Mérouane Debbah. Opportunistic Feedback Reporting and Scheduling Scheme for Multichannel Wireless Networks. GLOBECOM 2016 - 2016 IEEE Global Communications Conference, Dec 2016, Washington DC, United States. 10.1109/glocom.2016.7842075 . hal01373260

\section{HAL Id: hal-01373260 \\ https://hal.science/hal-01373260}

Submitted on 28 Sep 2016

HAL is a multi-disciplinary open access archive for the deposit and dissemination of scientific research documents, whether they are published or not. The documents may come from teaching and research institutions in France or abroad, or from public or private research centers.
L'archive ouverte pluridisciplinaire HAL, est destinée au dépôt et à la diffusion de documents scientifiques de niveau recherche, publiés ou non, émanant des établissements d'enseignement et de recherche français ou étrangers, des laboratoires publics ou privés. 


\title{
Opportunistic Feedback Reporting and Scheduling Scheme for Multichannel Wireless Networks
}

\author{
Matha Deghel ${ }^{\star, \diamond}$, Student, IEEE, Mohamad Assaad ${ }^{\star}$, Senior, IEEE, and Mérouane Debbah ${ }^{\diamond, \dagger}$, Fellow, IEEE \\ ${ }^{\star}$ Laboratoire de Signaux et Systèmes (L2S, UMR8506) CentraleSupélec-CNRS-Université Paris-Sud, Gif-sur-Yvette, France \\ ${ }^{\diamond}$ Large Networks and Systems Group (LANEAS), CentraleSupélec, Gif-sur-Yvette, France \\ ${ }^{\dagger}$ Mathematical and Algorithmic Sciences Lab, Huawei France R\&D, Paris, France \\ \{matha.deghel, mohamad.assaad, merouane.debbah\}@ centralesupelec.fr
}

\begin{abstract}
This work studies the problems of feedback allocation and scheduling for a multichannel downlink cellular network under limited and delayed feedback. We consider a realistic scenario where a fixed and small number $\bar{F}$ of link states can be reported to the base-station (BS) per time-slot. We study the trade-off between knowing at the BS a small number of accurate link states (i.e. that can be reported within one time-slot) and a larger but outdated number of link states (i.e. number of link states $>\bar{F}$ that requires more than one slot to be reported). We propose an efficient algorithm that selects the link states that should be reported to the base-station. A novelty here is that this feedback allocation algorithm is performed at the users side. We show that this algorithm combined with the MaxWeight scheduling achieves at least a fraction $\eta$ of the stability region achieved under the ideal system (i.e. with full and perfect feedback at no cost). We then provide numerical results that show the best aforementioned trade-off under various system setups.
\end{abstract}

\section{INTRODUCTION}

A powerful solution to improve the performance of wireless networks is user scheduling. This solution consists in allocating the resources (frequencies, slots) to the users based on their channel states. For such networks, an important factor to consider is the traffic pattern, meaning that each user has an incoming traffic process and a limited queue capacity. So the system stability (i.e. when all the queue lengths are finite) is an important property the scheduling mechanism should take into account. For these systems, Max-Weight (MW) scheduling policies were shown to be throughput optimal, that is to say, they provide optimal stability performances [1]. However, this latter work assumes that the scheduler has complete (i.e. perfect and full) knowledge of the channel states at each time-slot, where the cost of acquiring such information is not accounted for.

In realistic scenarios, however, complete feedback knowledge is not readily available at the scheduler. Different limitations result in such incomplete information, such as, for instance, the limited feedback and the delay. Scheduling under incomplete feedback knowledge has been investigated under various traffic and network scenarios [2]-[4]. The authors in [5] have examined three different scheduling approaches (centralized, decentralized and mixed) for multiple-input singleoutput (MISO) wireless downlink time-division duplex (TDD) system under zero forcing (ZF) precoding. In addition, under multiple-input multiple-output (MIMO) systems, the work in [6] studies the stability performance of a TDD wireless network using interference alignment (IA) technique under limited backhaul capacity and where the channel state information (CSI) acquisition cost is accounted for. Specifically, for this system, a precise characterization of the stability region is provided and a comparison with a TDMA-ZF system is investigated. Further, the authors of [7] investigate the impact of CSI quantization on the stability of a frequency-division duplex (FDD) system using ZF precoding technique. On the other hand, the work in [8] derives the optimal feedback scheme for a single-channel downlink system under partial channel state information. In [9], the authors study centralized scheduling with rate adaptation under imperfect channelestimator joint statistics. Throughput optimal scheduling with delayed feedback was investigated in [10]-[12]. In addition, the authors in [13] study the impact of limited feedback resources on the achievable stability region for a multichannel downlink system.

Contributions: We consider a multichannel wireless downlink network where both limited feedback resources and delayed feedback information are accounted for. Here, the term "channel" denotes a certain frequency band, whereas the term "link" refers to the wireless connection between a user and the base-station (BS) over a specific frequency band. Let $L$ be the total number of channels in the system. We adopt a setting where the feedback capacity per slot is limited. Specifically, only $\bar{F}$ link states (i.e. CSI) can be reported to the BS per time-slot. However, the system can decide to report an amount of feedback $F>\bar{F}$, thus the feedback process will require more than one time-slot in order to be accomplished. Since the feedback process directly impacts the scheduling mechanism, it is of great importance to design an efficient joint feedback reporting and scheduling algorithm. We first provide an algorithm that uses exactly $L$ feedback resources, where the feedback and scheduling decisions are done at the users side. Specifically, under the assumption of continuous time for contention, for each channel the users contend among each other and only one user reports its state, and thus this user will be scheduled for transmission over this channel. We show that this algorithm achieves a fraction $\eta$ of the stability region of the ideal system (i.e. full and perfect feedback knowledge at no cost). This algorithm assumes continuous 
time for contention, but since in practice we have discrete time that may lead to a collision between the users, we propose a second algorithm that uses a different approach and that imitates the first algorithm to a great extent. The second algorithm consists in having $F$ feedback resources and in letting the users decide whether they should send their CSIs or not, and then the BS performs scheduling over each channel using Max-Weight rule. One can notice the importance of the trade-off between having more $F$, which leads to a greater number of reported link states but which are more outdated, and having less $F$, which means a lower number of reported links but which are more accurate (i.e. less outdated). Finally, we provide numerical results that find the best trade-off (i.e. best $F$ ) under various system setups, where different values of users velocity are considered.

It is noteworthy to mention that two main points differentiate our context from previous works that consider limited feedback for multichannel systems. The first point is that we account for both delayed and limited feedback, where the delay depends on the amount of feedback resources. This is unlike for example the work in [13] where only limited feedback is considered without accounting for the impact of delay. The second point is the idea of letting the users be involved in the feedback decision since in our proposed algorithm the feedback allocation is done at the users side. This approach is dissimilar to the one adopted in [13] where the BS makes the feedback allocation.

The rest of this paper is organized as follows. Section II presents the system model. In Section III, we provide two joint feedback allocation and scheduling algorithms, and then we study the stability performances of these algorithms. Section IV is dedicated to numerical results and relevant discussions. Finally, conclusion remarks are given in Section V.

\section{System Model}

We consider an FDD cellular wireless network, with one base-station, $N$ mobile users and $L$ channels; e.g. LTEOFDMA system. Data packets to be transmitted from the basestation to the users are stored in $N$ separate queues at the basestation. Time is slotted with the slots of all users synchronized. Let $Q_{i}(t)$ denote the length of queue $i$ at the beginning of timeslot $t$, and let $\mathbf{Q}(t)=\left[Q_{1}(t), \ldots, Q_{N}(t)\right]$. This system can be seen as a multi-queue, multi-server, discrete-time queueing system. Note that since we work under an OFDMA-like system, at a given slot a channel can be allocated to one and at most one user.

We denote by $h_{i j}(t)$ the fading of the link connecting the base-station and mobile $i$ using channel $j$. The received signal for the $i$ th user if it gets scheduled on the $j$ th channel at timeslot $t$ is given as the following

$$
\sqrt{P} h_{i j}(t) x_{i j}(t)+n_{i j}(t),
$$

where $x_{i j}(t)$ is the corresponding complex-valued data stream, with $\mathbb{E}\left\{\left|x_{i j}\right|^{2}\right\}=1, P$ denotes the transmission power on each channel, and $n_{i j}(t)$ is the additive white Gaussian noise with zero mean and variance $\sigma_{n}^{2}$, i.e. $n_{i j}(t) \sim \mathcal{C N}\left(0, \sigma_{n}^{2}\right)$. The corresponding signal-to-noise ratio (SNR) is denoted and given as $\gamma_{i j}(t)=\rho\left|h_{i j}(t)\right|^{2}$, with $\rho=\frac{P}{\sigma_{n}^{2}}$. In any time-slot, the link state of a user on a channel represents the number of packets that can be successfully transmitted without outage to that user, on that channel. Transmission at a rate above the link state always fails, whereas transmission at a rate below or equal to the link state always succeeds. We use $C_{i j}(t)$ to represent the state of link $(i j)$ at time-slot $t$. We assume that each link state can take $K$ possible values $\left\{r_{1}, r_{2}, \ldots, r_{K}\right\}$, where rate $r_{k}$ represents state $k$ and corresponds to the case where $\tau_{k} \leq \mathrm{SNR}<\tau_{k+1}$, for some non-negative thresholds $\tau_{k}$. This setting is very used in practice where usually $K$ modulation and coding schemes are used (i.e. the instantaneous transmitted rate can take only $K$ possible values). For ease of exposition, we suppose that the $r_{k}$ are sorted in a descending order such that $r_{k}<r_{m}$ if $k>m$. We define $d$ to be the delay in number of time-slots. We use $\hat{h}_{i j}(t)$ to denote $h_{i j}(t-d)$, which is the fading of link $(i j)$ at time-slot $t-d$. We assume that in each slot, each user has a perfect estimation of the $h_{i j}, \forall j$, at this slot. For example, in time-slot $t-d$, user $i(\forall i)$ knows perfectly the $\hat{h}_{i j}(t)$ (i.e. $\left.h_{i j}(t-d)\right), \forall j$. The SNR corresponding to $\hat{h}_{i j}(t)$ is denoted and given by $\hat{\gamma}_{i j}(t)=\rho\left|\hat{h}_{i j}(t)\right|^{2}$. We use $\hat{C}_{i j}(t)$ to denote the state of link $(i j)$ at time-slot $t-d$, i.e. $\hat{C}_{i j}(t)=C_{i j}(t-d)$. Hence, $\hat{C}_{i j}(t)$ depends on the value of $\hat{\gamma}_{i j}(t)$, e.g. $\hat{C}_{i j}(t)=r_{k}$ if $\tau_{k} \leq \hat{\gamma}_{i j}(t)<\tau_{k+1}$.

We consider a realistic context where the feedback capacity per slot is limited. We assume that at most $\bar{F}$ link rates can be reported per slot. Therefore, if the system decides to report $F>\bar{F}$ link rates, a delay of $d$ slots is required, where $d=\left\lceil F(\bar{F})^{-1}\right\rceil$. One can see the problem that arises in this case: if more link states are reported to the BS, the Max-Weight has more CSIs that can be used in the decision, which improves the performance of the scheduling. On the other hand, a delay $d$ occurs between the estimation time of the CSIs and the scheduling decision time, and this delay increases with the number of reported link states, which results in performance reduction. The trade-off between the number of reported CSIs and the delay is a challenging problem that is considered at the end of this paper. Here, a necessary step to conduct the analysis is to specify/develop a joint feedback allocation and scheduling algorithm and to characterize its performance w.r.t. the ideal system where full and perfect CSI is available at no cost. This algorithm is designed in such a way as to greatly imitates another proposed algorithm that provides good stability performances but that is difficult to implement (because of an assumption of continuous time for contention). Since we consider a bursty traffic, the metric we use to evaluate the performance is the stability of the queues.

We assume that a transmission over link $(i j)$ can only be fulfilled if the corresponding $\hat{C}_{i j}(t)$ is reported to the BS. Let $\hat{Y}_{i j}(t)=Y_{i j}(t-d)$ denote the feedback decision associated with link $(i j)$ at time-slot $t-d$. So, we can write

$$
\hat{Y}_{i j}(t)= \begin{cases}1, & \text { if } \hat{C}_{i j}(t) \text { gets reported to the scheduler } \\ 0, & \text { otherwise }\end{cases}
$$


In addition, we define $S_{i j}(t)$ to be the scheduling decision associated with link $(i j)$ at time-slot $t$. So, we have

$$
S_{i j}(t)= \begin{cases}1, & \text { if user } i \text { gets scheduled on channel } j \\ 0, & \text { otherwise. }\end{cases}
$$

As mentioned earlier, in one time-slot, and on a given channel, one and at most one user can be scheduled. As a result, for all $t$ and all $j$, any valid service policy must obey $\sum_{i=1}^{N} S_{i j}(t) \leq 1$.

Each of the $N$ users has an incoming traffic process $A_{i}(t)$, which is an integer-valued process, measured in bits, i.i.d. (independent and identically distributed) in time and independent across users with $A_{i}(t)<A_{\max }$, for some finite constant $A_{\max }$. The mean rate of this process is $a_{i}=\mathbb{E}\left\{A_{i}(t)\right\}$. We assume that packets arrive at the BS at the beginning of the time-slot and are served at the end of the time-slot. Then, the queues in the system evolve according to the following equation For $1 \leq i \leq N$ :

$$
\begin{aligned}
& Q_{i}(t+1)= \\
& \left(Q_{i}(t)+A_{i}(t)-\sum_{j=1}^{L} \hat{C}_{i j}(t) \hat{Y}_{i j}(t) S_{i j}(t) \mathbb{1}_{\left(C_{i j}(t) \geq \hat{C}_{i j}(t)\right)}\right)^{+},
\end{aligned}
$$

where $\mathbb{1}_{(\cdot)}$ is the indicator function, the expression $\sum_{j=1}^{L} \hat{C}_{i j}(t) \hat{Y}_{i j}(t) S_{i j}(t) \mathbb{1}_{\left(C_{i j}(t) \geq \hat{C}_{i j}(t)\right)}$ represents the service rate allocated for user $i$ at time-slot $t$, and $(\cdot)^{+}=\max \{0, \cdot\}$. The use of indicator function $\mathbb{1}_{\left(C_{i j}(t) \geq \hat{C}_{i j}(t)\right)}$ is due to a possible mismatch between the reported rate, i.e. $\hat{C}_{i j}(t)$, and the actual link state, i.e. $C_{i j}(t)$, meaning that it is possible that the reported rate is greater than the actual link rate, thus leading to outage.

As mentioned previously, the adopted system has delayed and limited feedback information. On the other hand, for the ideal system all the $(N L)$ links feed their states back to the base station at no cost. That is to say, the BS receives an instantaneous (i.e. perfect) and full knowledge of the states of all the links in the system. For this latter system, it is straightforward to see that the service rate allocated for user $i$ at timeslot $t$ can be given by $\sum_{j=1}^{L} C_{i j}(t) S_{i j}(t)$ and, consequently, $Q_{i}(t+1)=\left(Q_{i}(t)+A_{i}(t)-\sum_{j=1}^{L} C_{i j}(t) S_{i j}(t)\right)^{+}$.

Finally, since we are interested in evaluating the stability performances of the system, we next recall the definitions of "strong stability" of a queuing system, "stability region" of a scheduling rule, and "Max-Weight" scheduling policy.

Definition 1 (Strong Stability). The condition for strong stability of the system can be expressed as

$$
\limsup _{T \rightarrow \infty} \frac{1}{T} \sum_{t=0}^{T-1} \mathbb{E}\left\{Q_{i}(t)\right\}<\infty, \forall i \in\{1, \ldots, N\},
$$

implying that the mean queue length of every queue is finite.

Definition 2 (Stability Region). The stability region a scheduling policy achieves can be defined as the set of vectors of mean arrival rates for which the system stays stable under this policy.
Definition 3 (Max-Weight Policy). In every time-slot, the Max-Weight rule schedules over channel $j$ the user that yields the largest product of queue length and link rate.

\section{Proposed Algorithm and Performance ANALYSIS}

In order for the base-station to perform scheduling, it needs to receive the rates of the links that were selected for the feedback. Hence, the feedback allocation process is of great importance since it directly impacts the scheduling mechanism. In this section, we first propose a joint feedback allocation and scheduling algorithm, named FSA, under which the allocations are done at the users side. We derive the fraction that this algorithm reaches of the stability region of the (ideal) system with perfect and full feedback knowledge. Although it provides good stability performances, this algorithm assumes continuous time for contention, which is not a realistic assumption since in practice the contention is in discrete time. For this reason, we propose a second algorithm, termed as FMA, that adopts another approach and that provides a good imitation of FSA. For FMA, an amount of $F$ feedback resources will be available, i.e. at most $F$ links can report their CSI to the base-station.

Let us use 'mdl' to represent the system where over each channel, only one user reports its feedback (and then uses this feedback to transmit after $d$ slots) and where this user is selected using algorithm FSA. We use 'pf' to denote the ideal system, i.e. perfect and full CSI is available at the BS, where MW policy is used to schedule users for transmission. Let $\mathcal{M}^{\mathrm{pf}}(t)$ and $\mathcal{M}^{\mathrm{mdl}}(t)$ be the subsets of scheduled links at time-slot $t$ under 'pf' and 'mdl', respectively.

We next provide the motivation behind the reasoning used in algorithms FSA and FMA.

Motivation: One could consider that the BS must decide of the feedback allocation. However, letting the users make this decision provides a gain due the following: if the BS decides which user should feed back its CSI, this decision will be based on the channel statistics. In other words, the BS can ask a user with bad CSI to report its feedback, since the BS cannot know beforehand if the current CSI is good or bad. However, the users estimate their CSI at each time-slot and therefore have perfect knowledge of their current CSIs. In addition, since we use the Max-Weight rule for scheduling which is affected by the feedback process, these users lack the queue lengths information. In general, the stability of the system is more sensitive to the channels variation than to the queues variation, so we can provide the users with the queue lengths knowledge every period of time and not every slot. This is done by the BS which broadcasts the queue lengths information every $T_{\mathrm{b}}$ slots, where $T_{\mathrm{b}}$ is typically high so that the (signalization) cost of such a broadcast stays negligible.

Algorithm 1 (FSA): For system 'mdl', the feedback and scheduling decisions are based on the following procedure.

1) Every $T_{\mathrm{b}}$ time-slots, that is to say, at time $0, T_{\mathrm{b}}, 2 T_{\mathrm{b}}, \ldots, n T_{\mathrm{b}}, \ldots$, the base-station broadcasts 
the queue lengths of all users, where $T_{\mathrm{b}}$ is typically high. So each user has outdated knowledge of the state of its queue (and all other queues). Let $\tilde{Q}_{i}(t)$ be the (outdated) queue length the users know at time $t$, i.e. $\tilde{Q}_{i}(t)=Q_{i}\left(n T_{\mathrm{b}}\right)$ for $t \in\left[n T_{\mathrm{b}},(n+1) T_{\mathrm{b}}[\right.$.

2) At time-slot $t-d$ :

To simplify the notation, we denote $\tilde{Q}_{i}(t-d)$ as $\hat{\tilde{Q}}_{i}(t)$. For each channel $j \in\{1, \ldots, L\}$, only one user sends its CSI to the BS. This user is the result of the Max-Weight rule [1], [10] and can be given by

$$
\arg \max _{i} \hat{\tilde{Q}}_{i}(t) \hat{C}_{i j}(t) \mathbb{P}\left\{C_{i j}(t) \geq \hat{C}_{i j}(t) \mid \hat{h}_{i j}(t)\right\} .
$$

To detect this user, we use an approach that consists in letting the users contend among each other for a certain period of time. Let $T_{\mathrm{c}}$ be the contention period for each channel. Assuming that contention can be done in continuous time [5], meaning that there is no collision between users, for each channel $j \in\{1, \ldots, L\}$ the contention is performed as the following:

User $i, \forall i \in\{1, \ldots, N\}$, waits until time

$$
T_{\mathrm{c}}\left(\hat{\tilde{Q}}_{i}(t) \hat{C}_{i j}(t) \mathbb{P}\left\{C_{i j}(t) \geq \hat{C}_{i j}(t) \mid \hat{h}_{i j}(t)\right\}\right)^{-1},
$$

then broadcasts a signal (of negligible duration). It is obvious that the user that broadcasts its signal first will be the result of (6) since this user will wait the smallest period of time. After the broadcast of the first signal, the contention procedure (of channel $j$ ) terminates and the corresponding user reports its CSI.

Once this procedure finishes, the contention of another channel gets started; clearly, two channels cannot have their contentions over the same period of time.

3) After getting the CSI (of time $t-d$ ) of each channel, the BS uses these CSIs for transmission at time-slot $t$. It is obvious that the user that will be selected to report its feedback (at time $t-d$ ) of channel $j$ will also be the user transmitting over this channel (at time $t$ ).

Note that here delay $d=\left\lceil L(\bar{F})^{-1}\right\rceil$ since FSA uses $L$ feedback resources. However, in FMA, $d=\left\lceil F(\bar{F})^{-1}\right\rceil$ because under this algorithm an amount of $F$ feedback resources will be used. The same notation is used in both algorithms for the sake of notational simplicity. As a result of algorithm FSA, at time-slot $t$, the expected weighted throughput, termed as $g_{\mathrm{mdl}}$, can be expressed as

$$
\begin{aligned}
& g_{\mathrm{mdl}}(\hat{\tilde{\mathbf{Q}}}(t))= \\
& \mathbb{E}\left\{\sum_{j=1}^{L} \sum_{i=1}^{N} \hat{\tilde{Q}}_{i}(t) \hat{C}_{i j}(t) \hat{Y}_{i j}(t) S_{i j}(t) \mathbb{1}_{\left(C_{i j}(t) \geq \hat{C}_{i j}(t)\right)} \mid \hat{\tilde{\mathbf{Q}}}(t)\right\} .
\end{aligned}
$$

Recall that $S_{i j}(t)$ represents the scheduling decision, so here $S_{i j}(t)=\hat{Y}_{i j}(t)$ and is equal to 1 if user $i$ is selected (at time $t-d$ ) by algorithm FSA to report its CSI (and consequently to transmit at time $t$ ) and 0 otherwise.
On the other hand, under the ideal system, the BS has full and non-delayed (i.e. perfect) knowledge of all the link states. Thus, at every time-slot, e.g. slot $t$, the Max-Weight policy schedules over channel $j$ user $i^{*}$ such that

$$
i^{*}=\arg \max _{i} Q_{i}(t) C_{i j}(t)
$$

which yields $S_{i^{*} j}(t)=1$; recall that $\sum_{i=1}^{N} S_{i j}(t) \leq 1$. For this system, we define $g_{\text {pf }}$ to be the expected weighted throughput, so we have

$$
g_{\mathrm{pf}}(\mathbf{Q}(t))=\mathbb{E}\left\{\sum_{j=1}^{L} \sum_{i=1}^{N} Q_{i}(t) C_{i j}(t) S_{i j}(t) \mid \boldsymbol{Q}(t)\right\}
$$

We now investigate the fraction that algorithm FSA can achieve w.r.t. the stability region of the ideal system. To this end, we define $\beta_{\mathrm{c}}$ as the following

$$
\beta_{\mathrm{c}}=\max _{t,(i j)}\left\{\frac{C_{i j}(t)}{\hat{C}_{i j}(t)}\right\} .
$$

Here we assume that all the possible values of $C_{i j}(t)$ (and thus of $\left.\hat{C}_{i j}(t)\right)$, given by $r_{1}, \ldots, r_{K}$, are different than zero. In practice, it is very rare not to be able to transmit at a nonzero rate. In addition, we define $p_{\mathrm{c}}^{\min }$ as

$$
p_{\mathrm{c}}^{\min }=\min _{(i j)} p_{\mathrm{c} i j}^{\min }
$$

with $p_{\mathrm{c} i j}^{\min }$ given by

$$
p_{\mathrm{c} i j}^{\min }=\min _{t, \hat{h}_{i j}(t)}\left\{\mathbb{P}\left\{C_{i j}(t) \geq \hat{C}_{i j}(t) \mid \hat{h}_{i j}(t)\right\}\right\},
$$

where we recall that the value of $\hat{C}_{i j}(t)$ depends on $\hat{h}_{i j}(t)$. Based on the above, we now provide the stability region that system 'mdl' can achieve compared with the stability region of the ideal system.

Theorem 1. Algorithm FSA can achieve at least a fraction $\eta$ of the stability region achieved by the ideal system (with perfect and full feedback), where

$$
\eta=\left(1-\frac{1}{T_{\mathrm{b}}}\right) \frac{p_{\mathrm{c}}^{\min }}{\beta_{\mathrm{c}}} .
$$

Proof. The detailed proof can be found in the extended version of the paper [14].

Based on the above result, we can state that algorithm FSA generally provides good stability performances. However, implementing this algorithm is a complicated task due to the assumption that the contention can be done in continuous time. This assumption is not realistic since in practice the contention is done in discrete time, leading to a possible collision between the users, and this problem is not handled in FSA. Thus, we next propose another algorithm that adopts a different approach and that provides a good imitation of FSA, and therefore good stability performances.

We use 'dl' to represent the system with delayed and limited feedback, and where the feedback and scheduling 
decisions are determined by algorithm FMA. Under FMA, the feedback allocation is done at the users side, and an amount of $F$ feedback resources is available. Specifically, under this algorithm, at time-slot $t-d$ the users decide to report at most $F$ link states. Let us denote the set of these links by $\hat{\mathcal{F}}(t)$, where the cardinality $|\hat{\mathcal{F}}(t)| \leq F$. Then, due to the delay of $d$ slots, the BS receives the totality of this feedback at time $t$. We recall that here $d=\left\lceil F(\bar{F})^{-1}\right\rceil$. Let us define $F_{i}(t-d)$ to be the number of link states user $i$ decides at time $t-d$ to report under FMA, so we have $\sum_{i=1}^{N} F_{i}(t-d) \leq F$. Also, we define $\alpha_{i}(t)$ to be some threshold that user $i$ updates with the time. To simplify the notation, we let $\hat{F}_{i}(t)$ and $\hat{\alpha}_{i}(t)$ represent $F_{i}(t-d)$ and $\alpha_{i}(t-d)$, respectively. Algorithm FMA is based on the idea of combining the Max-Weight and threshold-based concepts [10], [11]. It is worth mentioning that the steps in FMA are different from what is proposed in these latter works.

Algorithm 2 (FMA): For system 'dl', the feedback and scheduling decisions are based on the following procedure.

1) Every $T_{\mathrm{b}}$ time-slots, that is to say, at time $0, T_{\mathrm{b}}, 2 T_{\mathrm{b}}, \ldots, n T_{\mathrm{b}}, \ldots$, the base-station broadcasts the queue lengths of all users, where $T_{\mathrm{b}}$ is typically high. So each user has an outdated knowledge of the state of its queue (and all other queues). Let $\tilde{Q}_{i}(t)$ be the (outdated) queue length the users know at time $t$, i.e. $\tilde{Q}_{i}(t)=Q_{i}\left(n T_{\mathrm{b}}\right)$ for $t \in\left[n T_{\mathrm{b}},(n+1) T_{\mathrm{b}}[\right.$.

2) Each user knows then the queues of all other users and sorts all the queue lengths (including its queue) in a descending order. Let $i_{m}$ be the index of the user at the $m$ th position; e.g. $i_{1}$ is the index of the user with the largest queue length. A tie is broken by picking the user with the smallest index. We define $k\left(i_{m} j\right)$ as the state of link $\left(i_{m} j\right)$, so $\hat{C}_{i_{m} j}(t)=r_{k\left(i_{m} j\right)}$, where $k\left(i_{m} j\right) \in\{1, \ldots, K\}$.

3) At the beginning of time-slot $t-d$ :

To simplify the notation, we denote $\tilde{Q}_{i}(t-d)$ as $\hat{\tilde{Q}}_{i}(t)$. Set $\hat{F}_{i_{m}}(t)=0, \forall i_{m} \in\{1, \ldots, N\}$.

For $m=1$, which yields index $i_{1}$ and thus corresponds to the user with the largest queue length, the allocation process starts as the following:

a) For all $j \in\{1, \ldots, L\}$ :

Based on $r_{k\left(i_{m} j\right)}$, user $i_{m}$ sets $\hat{C}_{i_{m} j}(t)=r_{k\left(i_{m} j\right)}$.

- If there are enough feedback resources, i.e. if $\sum_{i_{m}=1}^{N} \hat{F}_{i_{m}}(t)<F$ :

* If channel $j$ satisfies the following inequality

$$
\hat{\tilde{Q}}_{i_{m}}(t) \hat{C}_{i_{m} j}(t) \mathbb{P}\left\{C_{i_{m} j}(t) \geq \hat{C}_{i_{m} j}(t) \mid \hat{h}_{i_{m} j}(t)\right\} \geq \hat{\alpha}_{i_{m}}(t)
$$

then $\hat{C}_{i_{m} j}(t)$ will be reported to the base-station and $\hat{F}_{i_{m}}(t)=\hat{F}_{i_{m}}(t)+1$.

* Else, $\hat{C}_{i_{m} j}(t)$ will not be reported.

- Otherwise, the allocation process stops since no enough feedback resources are available.

b) At this step user $i_{m}$ has just finished its part of the algorithm, so he broadcasts a special symbol to inform the BS that he finished the feedback allocation. Now, if the condition $\sum_{i_{m}=1}^{N} \hat{F}_{i_{m}}(t)<F$ is still satisfied and $m<N$, increment $m$ by 1 , and go to Step a). Otherwise, the allocation process stops.

4) At time-slot $t$, the BS receives the feedback that was selected at time-slot $t-d$. Then, for each channel, the BS applies the Max-Weight rule for scheduling. Specifically, over channel $j$, the BS schedules the user that results from the following:

$$
\arg \max _{i:(i j) \in \hat{\mathcal{F}}(t)} Q_{i}(t) \hat{C}_{i j}(t)
$$

We recall that if for some channel no feedback is reported, then no transmission will take place over this channel.

We now discuss the conditions that an algorithm should guarantee in order to provide a good imitation of FSA. To design an algorithm that greatly imitates FSA, two essential points should be accounted for. The first point is to ensure that over channel $j$ the user that will be scheduled is the result of

$$
\arg \max _{i} \hat{\tilde{Q}}_{i}(t) \hat{C}_{i j}(t) \mathbb{P}\left\{C_{i j}(t) \geq \hat{C}_{i j}(t) \mid \hat{h}_{i j}(t)\right\} .
$$

The second point is to make sure that a transmission occurs over each channel, which can be ensured by having at least one reported link state for each channel. This is necessary since FSA guarantees a reported link state for each channel, leading to a transmission over all the channels. Based on these two conditions, we can make the following remark.

Remark 1. Algorithm FMA can imitate algorithm FSA to a great extent. Hence, FMA, as FSA, generally provides good stability performances.

We now provide the motivation behind the statement in Remark 1. By allocating a sufficient amount of feedback resources, $F$, with $F$ sufficiently large, and using condition (14) which ensures the selection (for the feedback) of links with large value of $\hat{\tilde{Q}}_{i}(t) \hat{C}_{i j}(t) \mathbb{P}\left\{C_{i j}(t) \geq \hat{C}_{i j}(t) \mid \hat{h}_{i j}(t)\right\}$, we can claim that algorithm FMA guarantees the first point with high probability. On the other side, by a judicious choice of thresholds $\hat{\alpha}_{i}(t)$, the second point can be guaranteed. This can be explained as follows. We derive an upper bound on the probability of having a channel without a feedback, and we show that by carefully choosing the $\hat{\alpha}_{i}(t)$, this bound and consequently this probability can be made negligible. We denote the event of having channel $j_{1}$ (for all $j_{1} \in\{1, \ldots, L\}$ ) without a feedback by $\mathcal{E}_{j_{1}}$, i.e. this event occurs if $\left(i j_{1}\right) \notin$ $\hat{\mathcal{F}}(t), \forall i$. We recall that under FMA, a necessary condition for a link to be selected for the feedback is (see (14)):

$$
\hat{\tilde{Q}}_{i}(t) \hat{C}_{i j}(t) \mathbb{P}\left\{C_{i j}(t) \geq \hat{C}_{i j}(t) \mid \hat{h}_{i j}(t)\right\} \geq \hat{\alpha}_{i}(t) .
$$

Let $\mathcal{E}_{1, j_{1}}$ be the event that occurs if the above condition is satisfied for at least $F$ links $(i j)$, with $j \neq j_{1}$. We thus 
have $\mathcal{E}_{j_{1}}=\mathcal{E}_{1, j_{1}} \cap \mathcal{E}_{2, j_{1}}$, for some event $\mathcal{E}_{2, j_{1}}$. Hence, for the probability of $\mathcal{E}_{j_{1}}$, we have

$$
\begin{aligned}
& \mathbb{P}\left\{\mathcal{E}_{j_{1}}\right\}=\mathbb{P}\left\{\mathcal{E}_{1, j_{1}} \cap \mathcal{E}_{2, j_{1}}\right\} \leq \mathbb{P}\left\{\mathcal{E}_{1, j_{1}}\right\}= \\
& \mathbb{P}\left\{\sum_{\substack{(i j) \in \hat{\mathcal{F}}(t) \\
j \neq j_{1}}} \mathbf{1}_{\left.\left(\hat{\tilde{Q}}_{i}(t) \hat{C}_{i j}(t) \mathbb{P}\left\{C_{i j}(t) \geq \hat{C}_{i j}(t) \mid \hat{h}_{i j}(t)\right\} \geq \hat{\alpha}_{i}(t)\right) \geq F\right\} \stackrel{(a)}{\leq}}\right. \\
& \left(\frac{\exp (\delta)}{(1+\delta)^{(1+\delta)}}\right)^{\mu},
\end{aligned}
$$

in which $\delta=\frac{F}{\mu}-1$ and $\mu$ is the mean value of variable

$$
\sum_{(i j) \in \hat{\mathcal{F}}(t), j \neq j_{1}} \mathbf{1}_{\left(\hat{\tilde{Q}}_{i}(t) \hat{C}_{i j}(t) \mathbb{P}\left\{C_{i j}(t) \geq \hat{C}_{i j}(t) \mid \hat{h}_{i j}(t)\right\} \geq \hat{\alpha}_{i}(t)\right)},
$$

and where inequality (a) results from the Chernoff bound. Knowing $\hat{\tilde{Q}}_{i}(t), \hat{C}_{i j}(t)$, and $\mathbb{P}\left\{C_{i j}(t) \geq \hat{C}_{i j}(t) \mid \hat{h}_{i j}(t)\right\}$, user $i, \forall i \in\{1, \ldots, N\}$, can choose threshold $\hat{\alpha}_{i}(t)$ in such a way as to ensure that the bound in (18) is negligible. Consequently, the probability that a channel has no feedback, which is given by $\mathbb{P}\left\{\mathcal{E}_{j_{1}}\right\}$, will also be negligible. Therefore, the second point to ensure a good imitation of algorithm FSA can be guaranteed under FMA by carefully choosing the $\hat{\alpha}_{i}(t)$.

\section{NUMERICAL RESULTS AND DISCUSSIONS}

In this section we present our numerical results. We set $N=L=30, P=10 \log _{10}(10)=10 \mathrm{~dB}, \sigma_{n}=1$. We consider an LTE-like system with a bandwidth of $180 \mathrm{KHz}$ per channel and a carrier frequency $f_{\mathrm{c}}=2.1 \mathrm{GHz}$. Here we assume that a slot has a period of $T_{\mathrm{s}}=1 \mathrm{msec}$. To model the impact of delay, we consider the Gauss-Markov block fading process [15]. Then, we can write

$$
h_{i j}(t)=\sigma \hat{h}_{i j}(t)+e_{i j}(t),
$$

where $h_{i j}(t)$ is a complex normal random variable with zero mean and unit variance, i.e. $h_{i j}(t) \sim \mathcal{C N}(0,1)$, and where $e_{i j}(t) \sim \mathcal{C N}\left(0, \sigma_{e}^{2}\right)$ is the error due to delay. Notice that $\hat{h}_{i j}(t) \sim \mathcal{C N}(0,1)$ and $\sigma_{e}^{2}=1-\sigma^{2}$. The correlation coefficient is given by $\sigma=J_{0}\left(2 \pi f_{\mathrm{ds}} T_{\mathrm{s}} d\right)$ with Doppler spread $f_{\mathrm{ds}}$, where $T_{\mathrm{s}}$ is the slot duration, $d$ is the delay in number of time-slots, and $J_{0}(\cdot)$ is the zero-th order Bessel function of the first kind. The Doppler spread can be given by $f_{\mathrm{ds}}=\frac{f_{\mathrm{c}} v}{c}$, where $v$ is the user velocity and $c=3 \times 10^{8} \mathrm{~m} / \mathrm{sec}$ is the speed of light; we assume that all the users have the same velocity $v$. Hence, the correlation coefficient $\sigma=J_{0}\left(2 \pi f_{\mathrm{ds}} T_{\mathrm{s}} d\right)=$ $J_{0}\left(2 \pi \frac{f_{\mathrm{c}} v}{c} T_{\mathrm{s}} d\right)$. The channels are assumed to be i.i.d. across users and frequencies. The set of possible rates $\left\{r_{1}, \ldots, r_{K}\right\}$ is given in Table I. We suppose that all the users have Poisson incoming traffic with the same mean arrival rate $a$, i.e. $a_{i}=a$, $\forall i \in\{1, \ldots, N\}$.

To show the stability performance of the system, we plot the total average queue length for different values of mean arrival rates. It is worth mentioning that the point where the total average queue length begins to increase very steeply is the point where the system becomes unstable.
TABLE I

POSSIBLE RATES

\begin{tabular}{|l||c|c|c|c|c|} 
Rate (bits/slot) & 505.32 & 570.58 & 622.69 & 666.08 & 703.24 \\
\hline Rate (bits/slot) & 735.74 & 764.62 & 790.61 & 891.75 & 964.36
\end{tabular}

We provide four figures where in each one we consider a different value of the velocity, $v$. Specifically, in Fig. 1 to 4 we set $v=1,5,10$, and $50 \mathrm{~km} / \mathrm{hr}$, respectively. Each figure depicts the variation of total average queue length for different values of mean arrival rate under various system settings. The first setting is ideal system 'pf', i.e. the system with perfect and full feedback. The three remaining settings consider system 'dl', i.e. the system with delayed and limited feedback where algorithm FMA is used for feedback and scheduling decisions, for three different amounts of feedback resources $F=50,100$, and 150. We recall that delay $d$ depends on the value of $F$. Here we assume that $F=50,100$, and 150 result in $d=1$, 2 , and 3 slots, respectively.

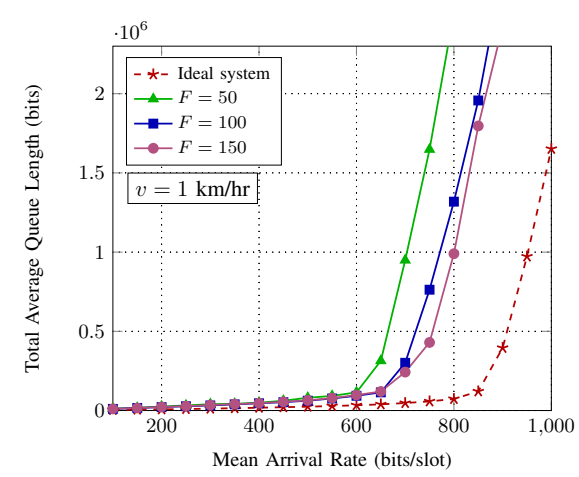

Figure 1: Total average queue length vs. mean arrival rate $a$. Here, the users velocity $v=1 \mathrm{~km} / \mathrm{hr}$.

The simulations show that for relatively small values of the velocity (Fig. 1 and Fig. 2), increasing the feedback resources $F$ to some limit can provide better stability performances. This is due to the fact that for small values of $v$, the variance of the error given by $\sigma_{e}^{2}=1-\sigma^{2}$ is sufficiently small, so the gain coming from having more feedback information overcomes the loss due to delay. Evidently, the limit until which if we increase $F$ we get better performances depends on $\sigma_{e}$ and consequently on $v$. It can be noticed that for $v=1 \mathrm{~km} / \mathrm{hr}$, the best $F$ we can choose is 150 . However, for $v=5 \mathrm{~km} / \mathrm{hr}$, the best $F$ is 100 . If we keep increasing $v$, there will be no gain from taking $F>50$. This can be seen in Fig. 3 and more clearly in Fig. 4 . In details, for $v=10 \mathrm{~km} / \mathrm{hr}$, setting $F=50$ (or $F=100$ ) yields better stability performances than the case where $F=150$. Also, for $v=50 \mathrm{~km} / \mathrm{hr}, F=50$ yields better performances than both of $F=100$ and $F=150$ cases. This is due to the fact that for relatively high velocities, the variance of the (delay) error, i.e. $\sigma_{e}^{2}$, is sufficiently high, so the loss due to delay is bigger than the gain coming from having more feedback information. 


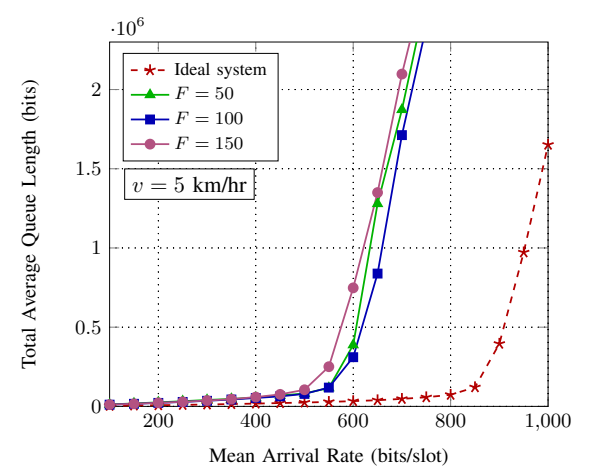

Figure 2: Total average queue length vs. mean arrival rate $a$. Here, the users velocity $v=5 \mathrm{~km} / \mathrm{hr}$.

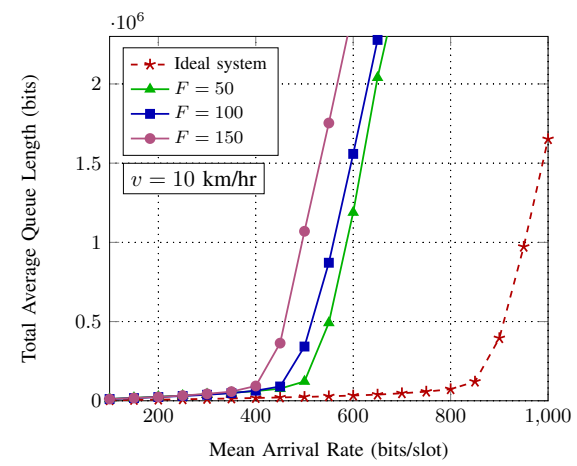

Figure 3: Total average queue length vs. mean arrival rate $a$. Here, the users velocity $v=10 \mathrm{~km} / \mathrm{hr}$.

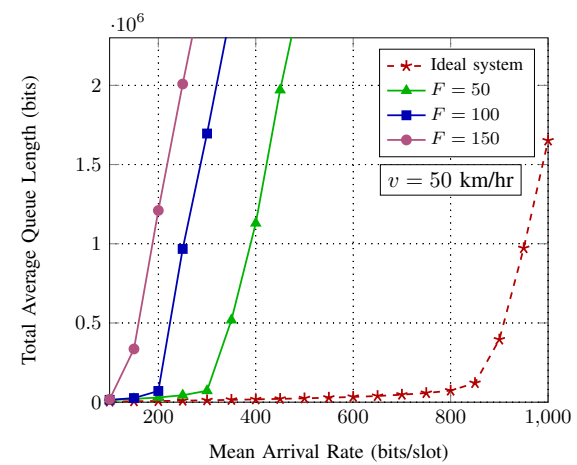

Figure 4: Total average queue length vs. mean arrival rate $a$. Here, the users velocity $v=50 \mathrm{~km} / \mathrm{hr}$.

\section{CONClusion}

In this work, we considered the feedback allocation and scheduling problems for a multichannel downlink cellular network under limited and delayed feedback. We first proposed an efficient joint feedback allocation and scheduling algorithm (FSA), in which the decisions are made at the users side. This algorithm is shown to achieve good stability performances with respect to the ideal system. However, it assumes that we have continuous time for contention, which is not a realistic assumption since in practice the contention is in discrete time. Thus, we proposed a second algorithm (FMA) that imitates the first one to a great extent and thus guarantees good stability performances, where the feedback decision is done at the users side and then the BS uses this feedback to perform users scheduling. Finally, under various system setups, i.e. different values of users velocity, we provided numerical results that find the best trade-off (i.e. best $F$ ) between having more feedback resources (thus, more knowledge at the scheduler) but longer delay (hence less accurate CSI) and having less feedback resources but low delay. Based on these same simulations, we noticed the good stability performances provided by using algorithm FMA.

We note that since under algorithm FMA the feedback allocation is performed at the users side and yields good stability performances, this suggests that the users must be more involved in the feedback allocation decision in future wireless networks.

\section{REFERENCES}

[1] L. Tassiulas and A. Ephremides, "Stability properties of constrained queueing systems and scheduling policies for maximum throughput in multihop radio networks," IEEE Transactions on Automatic Control, vol. 37, no. 12, pp. 1936-1948, Dec 1992.

[2] M. Assaad, "Frequency-time scheduling for streaming services in OFDMA systems," in 2008 1st IFIP Wireless Days, Nov 2008, pp. 1-5.

[3] A. Ahmad and M. Assaad, "Optimal resource allocation framework for downlink OFDMA system with channel estimation error," in 2010 IEEE Wireless Communication and Networking Conference, April 2010, pp. $1-5$.

[4] _ "Joint resource optimization and relay selection in cooperative cellular networks with imperfect channel knowledge," in Signal Processing Advances in Wireless Communications (SPAWC), 2010 IEEE Eleventh International Workshop on, June 2010, pp. 1-5.

[5] A. Destounis, M. Assaad, M. Debbah, and B. Sayadi, "Traffic-aware training and scheduling for MISO wireless downlink systems," IEEE Transactions on Information Theory, vol. 61, no. 5, pp. 2574-2599, May 2015.

[6] M. Deghel, M. Assaad, and M. Debbah, "Queueing stability and CSI probing of a TDD wireless network with interference alignment," in Information Theory (ISIT), 2015 IEEE International Symposium on, June 2015, pp. 794-798.

[7] K. Huang and V. Lau, "Stability and delay of zero-forcing SDMA with limited feedback," Information Theory, IEEE Transactions on, vol. 58, no. 10 , pp. 6499-6514, Oct 2012.

[8] A. Gopalan, C. Caramanis, and S. Shakkottai, "On wireless scheduling with partial channel-state information," IEEE Transactions on Information Theory, vol. 58, no. 1, pp. 403-420, Jan 2012.

[9] W. Ouyang, S. Murugesan, A. Eryilmaz, and N. B. Shroff, "Scheduling with rate adaptation under incomplete knowledge of channel/estimator statistics," in Communication, Control, and Computing (Allerton), 2010 48th Annual Allerton Conference on, Sept 2010, pp. 670-677.

[10] L. Ying and S. Shakkottai, "On throughput optimality with delayed network-state information," IEEE Transactions on Information Theory, vol. 57, no. 8, pp. 5116-5132, Aug 2011.

[11] A. A. Reddy, S. Banerjee, A. Gopalan, S. Shakkottai, and L. Ying, "Wireless scheduling with heterogeneously delayed network-state information," in Communication, Control, and Computing (Allerton), 2010 48th Annual Allerton Conference on, Sept 2010, pp. 1577-1584.

[12] M. Johnston and E. Modiano, "A new look at wireless scheduling with delayed information," in Information Theory (ISIT), 2015 IEEE International Symposium on, June 2015, pp. 1407-1411.

[13] M. Ouyang and L. Ying, "Approaching throughput optimality with limited feedback in multichannel wireless downlink networks," IEEE/ACM Transactions on Networking, vol. 21, no. 6, pp. 1827-1838, Dec 2013.

[14] M. Deghel, M. Assaad, and M. Debbah, "Extended version of the work on opportunistic feedback for multichannel wireless networks," 2016 [Online] http://goo.gl/6WGoLW.

[15] S. Haykin, Adaptive Filter Theory (3rd Ed.). Upper Saddle River, NJ, USA: Prentice-Hall, Inc., 1996. 